\section{Wnt signaling maintains the hair-inducing activity of the dermal papilla}

\author{
Jiro Kishimoto, Robert E. Burgeson, \\ and Bruce A. Morgan ${ }^{1}$
}

Cutaneous Biology Research Center, Massachusetts General Hospital and Harvard Medical School, Charlestown, Massachusetts 02129 USA

The formation of the hair follicle and its cyclical growth, quiescence, and regeneration depend on reciprocal signaling between its epidermal and dermal components. The dermal organizing center, the dermal papilla (DP), regulates development of the epidermal follicle and is dependent on signals from the epidermis for its development and maintenance. GFP specifically expressed in DP cells of a transgenic mouse was used to purify this population and study the signals required to maintain it. We demonstrate that specific Wnts, but not Sonic hedgehog (Shh), maintain anagen-phase gene expression in vitro and hair inductive activity in a skin reconstitution assay.

Received February 1, 2000; revised version accepted April 3, 2000.

Coordinated development of epithelium and mesenchyme during morphogenesis is often achieved by reciprocal signaling between mutually dependent organizing centers in each layer. Although feedback between these organizing centers helps maintain coordinated morphogenesis, it also complicates the experimental analysis of inductive interactions. The ability to purify the cellular constituents of these signaling centers and maintain their activity in isolation can greatly simplify the analysis of discrete signaling events in a complex feedback loop.

The hair follicle presents an attractive experimental system to study epithelial-mesenchymal interactions. The initial formation of the follicle during embryogenesis relies on many of the intercellular signaling molecules that mediate epithelial-mesenchymal interactions elsewhere in the embryo. Moreover, these same signaling molecules are expressed in the adult follicle and could mediate similar inductive interactions during the hair cycle. The adult hair follicle undergoes a cycle of hair growth (anagen) followed by a regression phase (catagen), a quiescent phase (telogen), and re-entry into anagen to generate a new hair shaft in the existing follicle (Hardy 1992). The hair shaft is derived from the epithelial matrix cells at the base of the follicle. However, a cluster of dermal cells ensheathed by the matrix

[Key Words: Wnt signaling; epithelial-mesenchymal interactions; dermal papilla; cell purification]

${ }^{1}$ Corresponding author.

E-MAIL bruce.morgan@cbrc2.mgh.harvard.edu; FAX (617) 726-4453. cells, known as the dermal papilla (DP), is thought to supply inductive signals required for hair outgrowth (Fig. 1A). Reciprocal signaling from the epidermis is required for the formation of the DP fo (for review, see Sengel 1976) and may also explain the coordinated morphological changes in epithelial and dermal components of the follicle observed during the hair cycle. Consistent with this hypothesis, DP cells lose specific gene expression and hair inductive activity when isolated from the follicular epithelium (Kishimoto et al. 1999).

It is difficult to demonstrate the inductive activity of the DP in the mature hair follicle directly. As shown in Figure 1, the DP is embedded in the ensheathing epidermal components of the follicle and is not amenable to physical manipulation. However, the inductive activity of DP cells can be revealed in a skin reconstitution assay (Kamimura et al. 1997). When keratinocytes and dermal cells are mixed and grafted to a nude mouse host, hair follicles are formed in the reconstituted skin if active DP cells are included in the mixture. In their absence, smooth, hairless skin is formed by the graft.

As a first step toward analysis of signaling to the DP, we have generated a transgenic mouse line that expresses green fluorescent protein (GFP) in these cells (Fig. 1B) (Kishimoto et al. 1999). The transgene is active during anagen but is shut off during catagen and telogen. Thus, its expression correlates with the presumed profile of inductive activity in the DP. Anagen DP cells from the skin of this mouse were purified to homogeneity using a fluorescence activated cell sorter and shown to retain hair inductive activity in the skin reconstitution assay. However, this inductive activity and GFP expression were rapidly lost in culture (Fig. 1C) (Kishimoto et al. 1999), suggesting that a factor normally supplied by epidermal cells is required to maintain DP cells in the anagen state. In the work reported here, we examine the ability of signaling molecules that are expressed in follicular epithelium to maintain isolated DP cells in an active, anagen state.

\section{Results and Discussion}

One likely candidate for the signal that maintains DP cells was Sonic hedgehog (Shh). It is expressed in the epidermal component of the hair follicle and is required for the maturation of the DP during embryonic development (St.-Jacques et al. 1998; Chiang et al. 1999). Exogenous Shh can also accelerate the transition from telogen to anagen during the hair cycle, although it has not been determined whether this reflects a direct action on DP cells (Sato et al. 1999). To further evaluate Shh signaling during the hair cycle, gene expression was examined in newborn mice where hair follicles are synchronized in anagen. In dissociated skin from newborn GFP transgenic mice, Shh mRNA is detected in the GFP negative population of sorted cells, which includes follicular epidermis (HF; Fig. 2A). Anagen DP cells do not express Shh (DP; Fig. 2A) but appear to respond to it based on the 

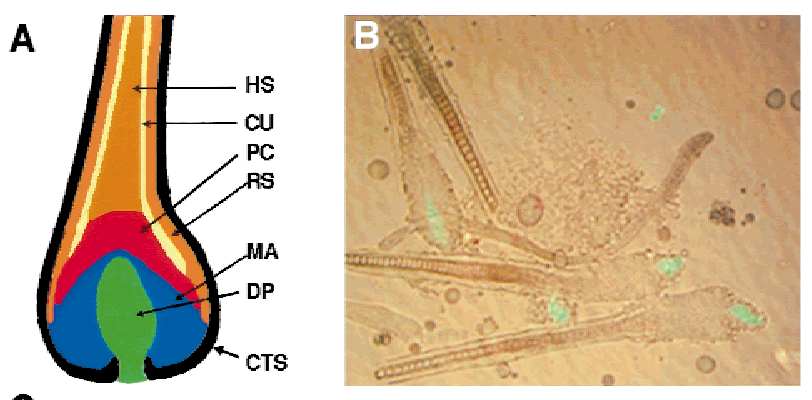

C

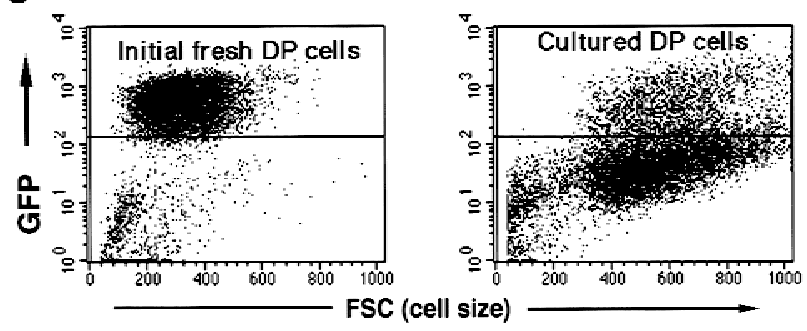

Figure 1. DP cells lose anagen-specific gene expression in culture. (A) Structure of the hair follicle. The dermal papilla (DP, green) is located at the bottom of the follicle, surrounded by undifferentiated epidermal matrix cells (MC, blue) that give rise to precursor cells (PC, red) and, ultimately, the differentiated hair shaft (HS, brown) and cuticle (CU, yellow). The hair shaft and DP are also surrounded by root sheath cells (RSC, orange) and the connective tissue sheath (CTS, black). (B) Isolated transgenic anagen hair follicles show GFP fluorescence in the DP. $(C)$ Flow cytometric analysis of GFP expression in DP cells immediately after isolation or after $90 \mathrm{hr}$ in culture. The horizontal line demarcates the gate defining GFP positive and negative subpopulations of DP cells.

levels of patched (ptc) and Gli1 found in freshly isolated DP cells. As a result of transcriptional feedback in the Shh signal transduction cascade, increased accumulation of mRNAs encoding ptc and Gli1 are observed in cells responding to Shh. This induction serves as an indication of response to the Shh signal (Goodrich et al. 1996; Marigo et al. 1996), and mRNAs from both genes are readily detected in freshly sorted DP cells (Fig. 2B). However, the abundance of both messages decreases to undetectable levels when isolated DP cells are cultured in the absence of follicular epithelium (P3; Fig. 2B). These findings suggest Shh signaling from follicular epithelium to the DP occurs in the anagen hair follicle and could cause DP activation in anagen.

We therefore evaluated whether Shh signaling was sufficient to rescue either GFP gene expression or hair inductive activity in DP cells maintained in culture. Freshly isolated DP cells were cocultured with either chick embryo fibroblasts (CEFs) that had been infected with a virus directing Shh expression or CEFs infected with a control vector. The use of heterospecific feeder cells allowed us to analyze gene expression in the DP cells by PCR with species-specific primers and to confirm that the DP cells received the Shh signal, as demonstrated by induction of both ptc and Gli1 (Fig. 3C). However, flow cytometric analysis revealed that GFP expression declined at identical rates in Shh-treated and

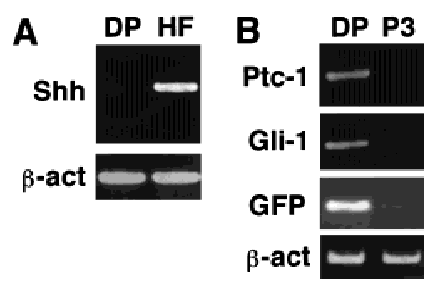

Figure 2. DP cells respond to Shh. (A) After dissociation of newborn skin, RT-PCR revealed Shh mRNA in the GFP-negative population $(\mathrm{HF})$ that includes the follicular epithelium. Shh mRNA was not detected in the GFP-positive population (DP). $\beta$-actin $(\beta$-act) serves to compare input RNA levels in this and subsequent panels. (B) ptc and Gli1 are expressed in freshly isolated DP cells, but transcript levels decrease upon passage in culture (P3), as do those from the GFP transgene.

control populations (Fig. 3A,B; data not shown). To confirm the correlation between GFP expression and maintenance of the anagen state, these cells were mixed with keratinocytes and grafted to nude mouse hosts. In this assay, the hairless skin that forms in the absence of ac-

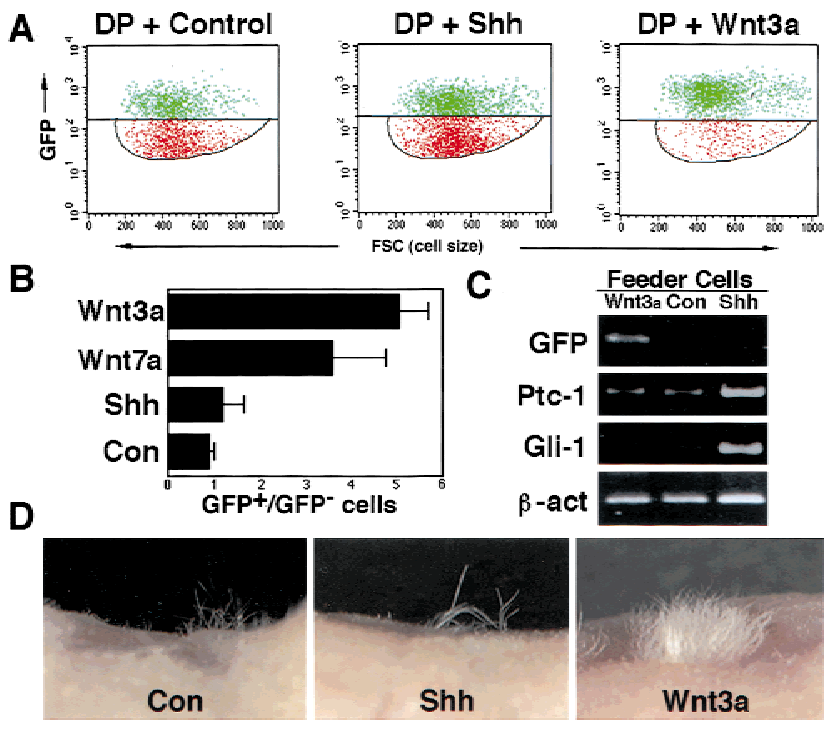

Figure 3. Wnt signaling to DP cells maintains hair inductive activity. (A) Flow cytometric analysis of DP cells cocultured with feeder cells producing Wnt3a or Shh, or infected with a control vector. The horizontal line demarcates the division between GFP-positive (green) and -negative (red) DP cells. The feeder cells found below the lower gate were excluded from this analysis. $(B)$ The ratio of GFP-positive to GFP-negative cells is shown for each treatment. (C) RT-PCR analysis of cocultured DP cells. GFP mRNA levels were maintained by exposure to Wnt3a but not Shh or control feeder layers. ptc and Gli1 expression was induced in DP cells cocultured with Shh-expressing feeder cells. DP cells cultured with control feeders that do not express Shh or feeders expressing Wnt3a express low levels of ptc and undetectable levels of Gli1. (D) Wnt3a-treated cells maintain hair inductive activity. Three weeks after grafting to nude mouse hosts, only occasional hairs were observed in grafts of control or Shh-treated DP cells. DP cells exposed to Wnt3a directed formation of a dense patch of hair in the graft. 
Table 1. Hair-forming potency of Shh-or Wnt-stimulated DP cells

\begin{tabular}{lrrr}
\hline Feeder cell & \multicolumn{3}{c}{ Hair induction $^{\mathrm{a}}$} \\
\hline Control & $1 / 13$ & $(0 / 5)$ & $8 \%$ \\
Shh & $2 / 10$ & $(1 / 4)$ & $20 \%$ \\
Wnt3a & $13 / 13$ & $(4 / 4)$ & $100 \%$ \\
\hline
\end{tabular}

${ }^{\mathrm{a} N u m b e r}$ of grafts that grew hair/total number of grafts performed.

Numbers in parentheses indicate grafts performed with DP cells that were reisolated from the feeder populations by FACS prior to grafting. Additional grafts were performed with the same number $\left(2 \times 10^{6}\right)$ of DP cells and accompanying feeder population. Results were indistinguishable from those with repurified DP populations, and feeder cells had no hair inductive activity when grafted without DP cells.

tive DP cells was observed whether or not the DP cells had been cultured in the presence of Shh (Fig. 3D; Table 1).

As in vivo experiments have suggested that Shh stimulates the transition from telogen to anagen, we also assessed whether Shh signaling was sufficient to activate isolated DP cells that had been maintained in culture until both GFP expression and hair inductive activity were lost. By these criteria, these cells are formally analogous to telogen DP cells. No increase in either GFP expression or hair inductivity was observed (data not shown). Thus, although Shh may initiate anagen in vivo, it is likely that it either requires a cooperating signal from the epidermis to induce the anagen state in DP cells or acts on the DP in part indirectly, possibly by induction of a secondary signal in the epidermis.

An alternative candidate to mediate signaling to the DP was suggested by a search of the sequence in the versican enhancer used to drive GFP expression in this transgenic line that revealed a Lef/TCF binding motif. This suggested that a Wnt might serve as the signal from the epidermis to activate gene expression in the DP, because the Lef/TCF family of DNA-binding proteins mediate the transcriptional effects of Wnt signaling through the $\beta$-Catenin pathway (for review, see Arias et al. 1999). Wnts are secreted glycoproteins that bind to Frizzled receptors. In a process dependent on Disheveled proteins, Frizzled receptor engagement inhibits the phosphorylation of $\beta$-Catenin by a complex including GSK3. This results in accumulation of $\beta$-Catenin protein and its translocation to the nucleus, where it can bind TCF and stimulate transcription from associated genes. The TCF binding site in the versican promoter appears to be required for GFP expression because no transgene expression was observed in 10 independent transgenic lines when this region was deleted from the expression construct (data not shown). Because deletions elsewhere in the construct had no effect on GFP expression in transgenic mice (data not shown), a Wnt is a strong candidate to mediate transgene induction during anagen.

Several Wnts are expressed in the hair follicle and could serve this function. Wnt $3 a$ and Wnt10b are expressed in the follicular matrix cells (St.-Jacques et al.
1998; Millar et al. 1999), and Wnt3a, Wnt4, Wnt5a, and Wnt7a transcripts were detected in the GFP negative population from dissociated skin that includes the follicular epithelia (Fig. 4A). Furthermore, anagen DP cells are likely to be capable of responding to Wnts, because they express components of the Wnt signal transduction cascade including frizzled7, disheveled2, GSK3 $\beta, \beta$-catenin, and Lef1 (Fig. 4B).

Feeder cells expressing Wnts $3 \mathrm{a}, 4,5 \mathrm{a}$, or $7 \mathrm{a}$ were used to test the effects of Wnt signaling on freshly isolated DP cells. Although several other Wnts are also expressed in the hair follicle, these were chosen because expression vectors with proven biological activity were available (Riddle et al. 1995; Kengaku et al. 1998; B.A. Morgan, unpubl.). Coculture with Wnts 3 a or 7 a resulted in maintenance of GFP fluorescence in the majority of the DP cells as demonstrated by FACS analysis and also increased the level of expression per cell (Fig. 3A,B). RTPCR confirmed that this reflects increased levels of GFP RNA rather than stabilization of the encoded protein (Fig. 3C). Wnt4-expressing cells also showed some ability to maintain GFP expression in cocultured DP cells but were less potent than cells expressing either Wnt3a or Wnt7a, whereas Wnt5a had no effect on DP GFP expression (data not shown).

It is possible that the observed effects on DP gene expression are caused by a secondary signal generated in the feeder cells in response to the Wnts they produce. However, direct activation of the $\beta$-Catenin signaling pathway in the feeder cells by infection with a virus encoding a truncated form of $\beta$-Catenin (Capdevila et al. 1998; Noramly et al. 1999) did not result in the produc-

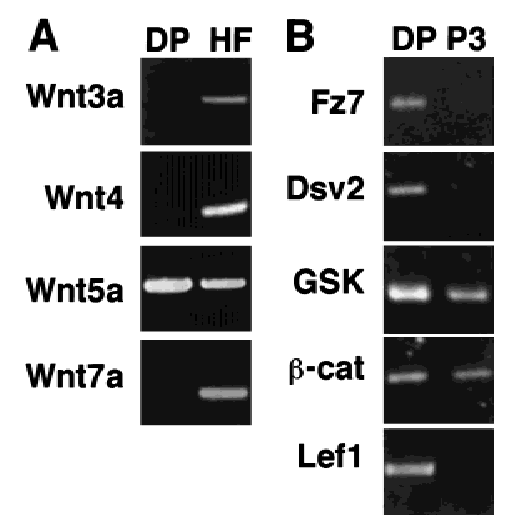

Figure 4. Expression of Wnts and their signal transduction cascade in the hair follicle. (A) Wnts 3a, 4, 5a, and $7 a$ were expressed in the GFP-negative population from the initial sort that includes follicular epithelial cells from anagen hair follicles $(\mathrm{HF})$. Note that the Wnt4 reaction was extended an additional six cycles to detect lower transcript levels. Of the Wnts analyzed, only Wnt5a was detected in the GFP positive population (DP). (B) Expression of genes encoding the Wnt signal transduction cascade was analyzed in DP cells immediately after isolation (DP) or after three passages in the absence of other cell types or exogenous factors (P3). frizzled7 $(f z 7)$, disheveled2 (dsv2), GSK3, $\beta$-catenin ( $\beta$-cat), and Lef1 were all expressed in freshly isolated DP cells. After three passages, expression of frizzled7, disheveled2, and Lef1 was reduced. 
tion of a signal that could maintain GFP expression in the DP cells (data not shown). This observation and the apparent requirement for a TCF binding site in the transgene enhancer suggest that Wnts act directly on the DP cells to maintain gene expression.

As suggested by GFP expression, the hair inductive activity of DP cells was also maintained in culture by Wnt signaling. When the murine DP cells were resorted, combined with keratinocytes, and used to reconstitute skin on a nude mouse host, Wnt3a- or Wnt7a-treated cells showed a dramatic increase in hair growth compared with cells cocultured with control feeders or Shhexpressing cells (Fig. 3D; Table 1; data not shown).

Although Wnt3a is sufficient to maintain DP cells in the anagen state, it cannot reactivate GFP expression or hair inductive activity in cells that have lost these properties in culture (data not shown). Analysis of transcripts encoding components of the Wnt signal transduction cascade reveals that Lef1, disheveled2, and frizzled7 are all down-regulated after maintenance in culture in the absence of Wnts (Fig. 4B). This could explain the failure of exogenous Wnt to reactivate GFP expression in these cells. Maintenance of the expression of proteins required for Wnt signal transduction by other factors expressed locally in the epidermis could contribute to the coordination of morphogenesis in the follicle.

The work reported here might predict that exogenous Wnt would extend the hair cycle and promote hair growth. However, previous experiments that forced the expression of Wnt3a or Disheveled2 in murine skin resulted in reduced hair growth (Millar et al. 1999). In those experiments, the transgenes were driven by the keratin 14 promoter/enhancer that drives expression in the distal outer root sheath (see Fig. 1A). It is thus unlikely that the DP cells were exposed to the exogenous Wnt. The fact that the cell autonomous activity of exogenous disheveled in the outer root sheath resulted in a similar phenotype suggests that the phenotypes caused by exogenous Wnt3a also resulted from autocrine Wnt signaling in this layer. That work, experiments manipulating $\beta$-Catenin levels in the epidermis of mice and chicks, and analysis of a reporter transgene driven by TCF binding sites have all have suggested a role for Wnt signaling in the epidermal component of the follicle (Gat et al. 1998; DasGupta and Fuchs 1999; Noramly et al. 1999|. The findings reported here demonstrate a role for Wnts in signaling to the DP and suggest the possibility that a single Wnt expressed in the epidermis could coordinate development in both the dermis and epidermis.

Our results demonstrate that Wnt3a and Wnt7a can act as inductive signals to maintain the DP in an anagen state. Although these Wnts are expressed in the epidermal component of the hair follicle and are candidates to mediate this activity in vivo, it is possible that another Wnt acting through the same signal transduction pathways may normally serve this function during the hair cycle. These results also suggest that Shh signaling is not sufficient to initiate or maintain the anagen state of DP cells and may therefore act at least in part indirectly to promote the transition to anagen in vivo. Finally, these results extend the correlation in these transgenic DP cells between GFP expression and the ability to induce hair growth. This approach will continue to prove a powerful tool to dissect signaling between epithelia and mesenchyme to coordinate morphogenesis.

\section{Materials and methods}

RT-PCR analysis

Total RNA was isolated, and RT-PCR was performed as described using an annealing temperature of $58^{\circ} \mathrm{C}$ (Kishimoto et al. 1999). The primers used are listed below. With the exception of Wnts 4, 5, and 7, primers were designed to distinguish between mouse and chicken orthologs, and the lack of cross reaction to chick sequences was confirmed on cDNA from feeder cells alone. Parentheses indicate the number of cycles used for each reaction to achieve semiquantitative amplification. Wnt3a, GCGCCCTGGCTCACTAC and ATGCTGCTGCTG CTGGCC (30); Wnt4, TGATCCAGAGGCAGGTGCAG and CTTCTCCAGTTCTCCACTGC (36); Wnt $5 a$, CTGTTGACCTGCACCAGCTT and TCAAGGAATGCCAGTACCAGTACCAG $(30)$; Wnt7 $a$, GCGAGCATCATCTGTAACAAG and AGCCACAGTCGCTCAGGTTG (30); frizzled7, CTGCTAGAGGACCGTGCC and AGGTGCGTTCCCAGTGCT (36); disheveled2, CATCCTTCAGCAGTGTCA and CGTCATTGTCATTCAGAG (36); GSK3 $\beta$, CAGGGCACCAGAGTTGAT and GCAGAAGCGGCGTTATTG $(30) ; \beta$-catenin; CCACCAGCTAGGCGCACT and GGGCTCAGAGGGTCCGAG (30); LEF1, ACTGTCAGGCGACACTTCC and TGCACGTTGGGAAGGAGC (36); Shh $(30)$; Ptc, AGCCTCACAGTAACACCC and TGTTCTCCTCCAGCATGA (36); Gli1, TTGGGGATGCTGGATGGG and CGGTCACTGGCATTGCTA (36); $\beta$-actin 5 '-CCACACCCGCCACCAGTTC-3' and 5'-GAGGAAGAGGATGCGGCA-3' (26); GFP as described (Kishimoto et al. 1999) (26).

\section{Cell culture}

Fresh mouse pelage DP cells were obtained from versican GFP transgenic newborn skin by high-speed cell sorting (MoFLO) as described previously (Kishimoto et al. 1999). CEFs were infected with RCASBP(A) retroviral vectors encoding chick Shh (Riddle et al. 1993), Wnt3a (Kengaku et al. 1998), Wnt4, Wnt5a (S. Noramly and B. Morgan, in prep.), or Wnt7a (Riddle et al. 1995), or vector alone as described (Morgan and Fekete 1996). GFP-positive mouse DP cells were added onto $30 \%-50 \%$ confluent feeder cells to achieve a ratio of 1:3 (mouse/chick). For the flow cytometric analysis, cells were cocultured in 24-well dishes for 48-96 hr. For the grafting experiment, cocultured cells were passaged two to three times in $10-\mathrm{cm}$ dishes to generate the $2 \times 10^{6}$ cells required for each graft.

Flow cytometry

Cells were trypsinized and resuspended in $0.5 \mathrm{ml}$ of PBS with $1 \%$ BSA. Flow cytometric analysis was performed by FACScan (Beckton-Dickinson). DP cells were labeled with mouse MHC class II monoclonal antibody and incubated with secondary IgG conjugated to PE. The PE-negative avian cells fell below the lower gate in Figure 3 and were excluded from the analysis of GFP expression.

Reconstitution assay

The reconstitution assay was performed as described (Kishimoto et al. 1999). Primary keratinocytes were prepared from two newborn pups per graft and combined with $2 \times 10^{6} \mathrm{DP}$ cells in the graft chamber. Hair growth was monitored 2 weeks after grafting and weekly thereafter.

\section{Acknowledgments}

We thank S. Jiang for cell sorting, S. Noramly for discussion of unpublished data, and H. Baden, L. Raftery, and C. Hunter Brunken for comments on the manuscript. This work was supported by a grant from Shiseido, Inc., Japan, to the CBRC, B.A.M. and R.E.B.

The publication costs of this article were defrayed in part by payment of page charges. This article must therefore be hereby marked "advertisement" in accordance with 18 USC section 1734 solely to indicate this fact.

\section{References}

Arias, A.M., A. Brown, and K. Brennan. 1999. Wnt signaling: Pathway or network? Curr. Opin. Genet. Dev. 9: 447-454. 
Capdevila, J., C. Tabin, and R. Johnson. 1998. Control of dorsoventral somite patterning by Wnt-1 and beta-catenin. Dev. Biol. 193: 182194.

Chiang, C., R.Z. Swan, M. Grachtchouk, M. Bolinger, Y. Litingtung, E.K. Robertson, M.K. Cooper, W. Gaffield, H. Westphal, P.A. Beachy, and A.A. Dlugosz. 1999. Essential role for Sonic hedgehog during hair follicle morphogenesis. Dev. Biol. 205: 1-9.

DasGupta, R. and E. Fuchs. 1999. Multiple roles for activated LEF/TCF transcription complexes during hair follicle development and differentiation. Development 126: 4557-4568.

Gat, U., R. DasGupta, L. Degenstein, and E. Fuchs. 1998. De novo hair follicle morphogenesis and hair tumors in mice expressing a truncated beta-catenin in skin. Cell 95: 605-614

Goodrich, L., R. Johnson, L. Milenkovic, J. McMahon, and M. Scott. 1996. Conservation of the hedgehog/patched signaling pathway from flies to mice: Induction of a mouse patched gene by Hedgehog. Genes \& Dev 10: 301-312.

Hardy, M.H. 1992. The secret life of the hair follicle. Trends Genet. 8: $55-61$.

Kamimura, J., D. Lee, H. Baden, J. Brissette, and G. Dotto. 1997. Primary mouse keratinocyte cultures contain hair follicle progenitor cells with multiple differentiation potential. J. Invest. Dermatol. 109: 534540.

Kengaku, M., J. Capdevila, C. Rodriguezesteban, J. Delapena, R.L. Johnson, J.C.I. Belmonte, and C.J. Tabin. 1998. Distinct Wnt pathways regulating AER formation and dorsoventral polarity in the chick limb bud. Science 280: 1274-1277.

Kishimoto, J., R. Ehama, L. Wu, S. Jiang, N. Jiang, and R.E. Burgeson. 1999. Selective activation of the versican promoter by epithelial-mesenchymal interactions during hair follicle development. Proc. Nat. Acad. Sci. 96: 7336-7341.

Marigo, V., M. Scott, R. Johnson, L. Goodrich, and C. Tabin. 1996. Conservation in hedgehog signaling: Induction of a chicken patched homolog by Sonic hedgehog in the developing limb. Development 122: $1225-1233$

Millar, S.E., K. Willert, P.C. Salinas, H. Roelink, R. Nusse, D.J. Sussman and G.S. Barsh. 1999. WNT signaling in the control of hair growth and structure. Dev. Biol. 207: 133-149.

Morgan, B. and D. Fekete. 1996. Manipulating gene expression with replication-competent retroviruses. In Methods in cell biology (ed. M. Bonner-Fraser), pp.185-218. Academic Press, San Diego, CA.

Noramly, S., A. Freeman, and B. Morgan. 1999. $\beta$-catenin signaling can initiate feather bud development. Development 126: 3495-3507.

Riddle, R., R. Johnson, E. Laufer, and C. Tabin. 1993. Sonic hedgehog mediates the polarizing activity of the ZPA. Cell 75: 1401-1416.

Riddle, R., M. Ensini, C. Nelson, T. Tsuchida, T. Jessell, and C. Tabin. 1995. Induction of the LIM homeobox gene Lmxl by WNT7a establishes dorsoventral pattern in the vertebrate limb. Cell 83: 631-640.

Sato, N., P.L. Leopold, and R.G. Crystal. 1999. Induction of the hair growth phase in postnatal mice by localized transient expression of Sonic hedgehog. J. Clin. Invest. 104: 855-864.

Sengel, P. 1976. Morphogenesis of skin. Cambridge University Press, Cambridge, UK.

St.-Jacques, B., H.R. Dassule, I. Karavanova, V.A. Botchkarev, J. Li, P.S Danierlian, J.A. McMahon, P.M. Lewis, R. Paus, and A.P. McMahon 1998. Sonic hedgehog signaling is essential for hair development. Curr. Biol. 8: 1058-1068. 


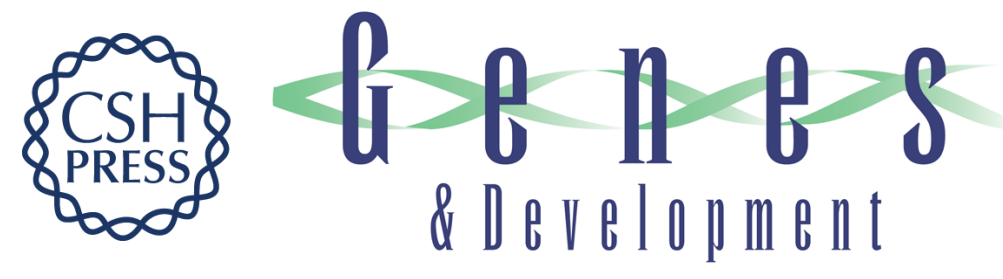

\section{Wnt signaling maintains the hair-inducing activity of the dermal papilla}

Jiro Kishimoto, Robert E. Burgeson and Bruce A. Morgan

Genes Dev. 2000, 14:

Access the most recent version at doi:10.1101/gad.14.10.1181

References This article cites 17 articles, 6 of which can be accessed free at: http://genesdev.cshlp.org/content/14/10/1181.full.html\#ref-list-1

License

Email Alerting Receive free email alerts when new articles cite this article - sign up in the box at the top Service right corner of the article or click here.

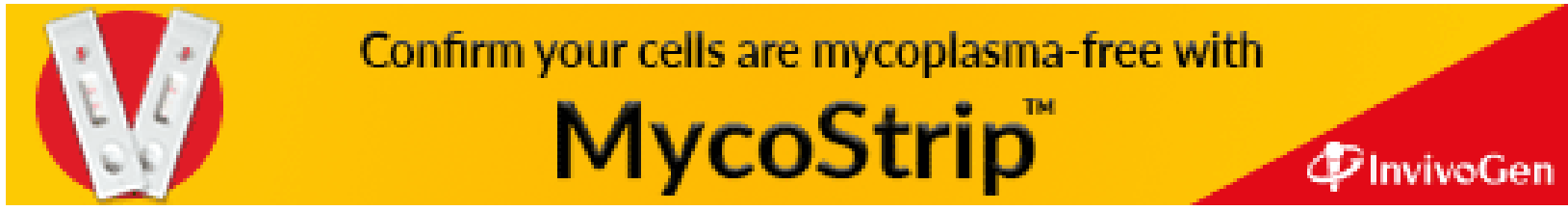

